\section{Acetato de Goserelin (Zoladex) más ablación endometrial para el sangrado uterino disfuncional: Un gran estudio doble ciego, aleatorizado.}

Jacques Donnez, M.D., Ph.D.

George Vilos, M.D.§

Michael J. Gannon, M.D. II

Søren Stampe-Sorensen, M.D.II

Ingemar Klinte, M.D.**

Robert M. Miller, M.D. $\dagger \dagger$

$¥$ Service de Gynecologie, University St. Lue, Brussels, Belgium.

$\S$ St. Joseph's Health care Center.

II Department of Obstetrics and Gynaecology. Leeds General Infirmary.

II K.A.S. Glostrup. Gynaekologisk Afd. G.

** Kvinnokliniken, Bassjukhuset.

t† Department of Medical Affairs, Zeneca Pharmaceuticals.

Fertil Steril 1997; 68: 29-36.

Objetivo: Confirmar las ventajas del goserelin mas la ablación endometrial para el tratamiento de la hemorragia uterina disfuncional.

Diseño: Estudio doble ciego, aleatorizado, prospectivo, multicéntrico.

Pacientes: Mujeres premenopáusicas con ciclos menstruales con sangrado uterina disfuncional.

Tratamiento: Las pacientes fueron aleatorizadas a goserelin o placebo (depósito falso) una vez al mes por dos meses antes de la ablación endometrial. El tratamiento fue calculado para permitir la cirugía 6 semanas después en el día 7 del ciclo menstrual.

Medidas principales de resultado: tasas de amenorrea, grosor e histología del endometrio, puntajes de dolor y de pérdida de sangre y parámetros quirúrgicos.

Resultados: A las 24 semanas después de la cirugía, experimentaron amenorrea, significativamente mas pacientes del grupo de goserelin que del de placebo $(40 \%$ versus $26 \%$ ). La pérdida de sangre fue menor que la basal, pero sin diferencias entre los grupos. En la cirugía el grosor endometrial fue de $1.6 \mathrm{~mm}$ y $3.4 \mathrm{~mm}$, para los grupos de goserelin y placebo, respectivamente, con glándulas y estroma significativamente mas atróficos en el grupo de goserelin. La cirugía fue significativamente mas corta (en $22 \%$ ) y mas fácil en el grupo de goserelin que en el de placebo, con una mediana de absorción de líquidos significativamente menor en el grupo de goserelin. En ambos grupos se redujeron los puntajes de dolor, la satisfacción fue alta (>92\%) y la tasa de reintervención fue baja $(2.8 \%)$.

Conclusión: El goserelin en combinación con la ablación endometrial fue superior a la ablación endometrial sola para el tratamiento de la hemorragia uterina disfuncional.

\section{Análisis cromosómico de fetos abortados de mujeres con aborto habitual positivas para anti- cuerpo anticardiolipina}

Koichi Takakuwa M.D.*†

Kensaku Asano, M.D.*

Masato Arakawa, M.D.*

Masako Yasuda, M.D.

Isao Hasegawa, M.D.*

Kenichi Tanaka, M.D.*

* Department of Obstetrics and Gynecology, Niigata University School of Medicine.

† Department of Obstetrics and Gynecology, 1-757, Asahimachi-dori, Niigata 951, Japan.

* Department of Obstetrics and Gynecology, Nagaoka Red Cross Hospital, Nagaoka, Japan.

Fertil Steril 1997; 68: 54-58.

Objetivo: Aclarar la relación entre el anticuerpo anticardiolipina y el aborto recurrente.

Diseño: Estudio clínico prospectivo

Lugar: Práctica institucional en la cual las pacientes con aborto recurrente fueron registradas en la clínica ambulatoria para infertilidad en el Hospital universitario Niigata.

Pacientes: quinientas sesenta y un pacientes con aborto recurrente y 148 pacientes que no eran abortadoras habituales y habían tenido un aborto espontáneo.

Intervención: Fueron recolectados de las pacientes los fetos abortados para análisis cromosómico.

Medidas principales de resultado: En las pacientes con aborto recurrente fue evaluada la tasa positiva de anticuerpos anticardiolipina. El análisis cromosómico fue realizado en 10 pacientes con anticuerpos anticardiolipina positivos quienes tuvieron otro embarazo que terminó en aborto repetido. Se realizaron análisis similares de fetos abortados de 148 mujeres control con abortos esporádicos tempranos.

Resultados: La incidencia de anormalidades cromosómicas en las abortadoras recurrentes con anticuerpo anticardiolipina positivo fue $20 \%$ ( 2 de 10 casos), el cual fue significativamente menor que en las pacientes con aborto esporádico $(60.1 \%, 89$ de 148 casos). 